\title{
On Key Reinstallation Attacks over 4G LTE Control-Plane: Feasibility and Negative Impact
}

\author{
Muhammad Taqi Raza, Yunqi Guo, Songwu Lu, and Fatima Muhammad Anwar
}

\begin{abstract}
ACM Reference Format:
Muhammad Taqi Raza, Yunqi Guo, Songwu Lu, and Fatima Muhammad Anwar . 2021. On Key Reinstallation Attacks over 4G LTE Control-Plane: Feasibility and Negative Impact. In Annual Computer Security Applications Conference (ACSAC '21), December 6-10, 2021, Virtual Event, USA. ACM, New York, NY, USA, 10 pages. https://doi.org/10.1145/3485832.3485833
\end{abstract}

\begin{abstract}
This paper studies the feasibility of key reinstallation attacks in the 4G LTE network. It is well known that LTE uses session keys for confidentiality and integrity protection of its control-plane signaling packets. However, if the keys are not updated and counters are reset, key reinstallation attacks may arise. In this paper, we show that several design choices in the current LTE security setup are vulnerable to key reinstallation attacks. Specifically, on the control plane, the LTE security association setup procedures, which establish security between the device and the network, are disconnected. The keys are installed through one procedure, whereas their associated parameters (such as uplink and downlink counters) are reset through another different procedure. The adversary can thus exploit the disjoint security setup procedures, and launch the key stream reuse attacks. He consequently breaks message encryption, when he tricks the victim to use the same pair of keys and counter value to encrypt multiple messages. This control-plane attack hijacks the location update procedure, thus rendering the device to be unreachable from the Internet. Moreover, it may also deregister the victim from the LTE network. We have confirmed our findings with two major US operators, and found that such attacks can be launched with software-defined radio devices that cost about \$299. We further propose remedies to defend against such threats.
\end{abstract}

\section{INTRODUCTION}

The current fourth-generation (4G) Long Term Evolution (LTE) technology provides billions of users their daily mobile Internet access. Different from the wired Internet, LTE has made security a top design goal, thus deploying several built-in security mechanisms. Together, such procedures provide all key security functions of authentication, encryption, integrity and access control.

In this paper, we examine the LTE security from a new perspective. It is well known that, the encryption and integrity protection components in LTE use mature and well-tested crypto algorithms that have been used for decades. Therefore, it seems that neither exhibits vulnerabilities. Motivated by the recent efforts on key reinstallation threats over wireless $[11,16,35,40]$, we hypothesize that

(C)
This work is licensed under a Creative Commons Attribution International
4.0 License.
ACSAC '21, December 6-10, 2021, Virtual Event, USA
(c) 2021 Copyright held by the owner/author(s).
ACM ISBN 978-1-4503-8579-4/21/12.
https://doi.org/10.1145/3485832.3485833

LTE may suffer from similar vulnerabilities. Indeed, our findings confirm the hypothesis. However, the threats are exposed via completely different procedures. The impacts are also more damaging.

Specifically, we study LTE security key installation method and counters handling process for a number of LTE procedures (such as device registration, deregistration, location update and others). 4G (like 3G) employs Authentication and Key Agreement (AKA) protocol to install the security keys and enables the integrity protection of its signaling messages. After that it runs Security Mode Command procedure to activate ciphering of messages at LTE subscriber. LTE employs stream ciphers which have been a popular method of encryption for the confidentiality of its signaling and data packets. The ciphering algorithm takes key (installed through AKA procedure), counter value and a couple others as an input and generates keystream block. The keystream block, $k$ is exclusive-ored (xored) with the plaintext message, $m$, to produce the encrypted message, $k \oplus m=e$. In practice, the keystream is pseudo random that generates the cipher text known as a one-time pad, proved unbreakable by Shannon[31]. It is an established fact that the security of stream ciphers rests on never reusing the keystream block $k$ [15]. In case $k$ is reused to cipher two different plaintext messages, $m$ and $n$, then the encrypted texts $k \oplus m$ and $k \oplus n$ can be xored together to recover $m \oplus n$. By using chosen-text attack, one can further break $m \oplus n$, and gets the messages $m$ and $n$.

The scenario in which LTE ciphering algorithm gives same keystream block over multiple rounds is the one in which the ciphering key remains constant and the counter value (responsible of generating random keystream block) is reset. We call this "key reinstallation" vulnerability. In this paper, we look LTE control-plane procedures that lead to key reinstallation attacks.

The idea behind our control-plane attacks can be summarized as follows. In the security establishment procedure, the device first installs new key through authentication procedure. Once the key is installed, the network runs security mode command procedure to reset the counter values for encryption. In reality, the signaling message may be lost or dropped. In case, the device response to security mode command request is dropped, the network reinitiates the security mode command procedure. On receiving the replayed security mode command request from the network, the device resets the counter values again before generating the response message. This means two signaling messages sent after two security mode command responses are encrypted with same keystream block at device. We show that an attacker can force count resets by blocking the response to security mode command request. By intentionally forcing count resets, the confidentiality protocol can be attacked, e.g., packets can be replayed, decrypted, and/or forged. The attacker can launch attacks on device location update and deregistration procedures. These attacks render the victim device to be unreachable from the outside world (e.g. it cannot receive voice 
calls), or even leaves the device without LTE service (i.e. no service scenario).

It is worth noting that our attacks do not violate the security properties proven in formal LTE analysis work, such as LTEInspector [14]. The formal method proofs state that LTE key should not be shared over the air, and all protocols behave as desired by the 3GPP standard. Our attacks do not leak ciphering or integrity keys and strictly follow LTE standards. Further, although the attacker can launch the attacks by reseting the counts, in control-plane he cannot repeatedly do so for more than one signaling message as the integrity protection becomes mandatory thereafter. However, this is sufficient for an attacker to launch as serious an attack as deregistering the victim subscriber from the LTE network.

In our experiments, we have verified all attack steps through two major US LTE operators. We use Software Defined Radio (SDR) to conduct our proof-of-concept studies. The experimental results show that LTE key reinstallation attacks are practical and pose a realistic threat to the LTE users. Last, we propose 3GPP standardcompliant remedies to address the discussed vulnerabilities. We prototype our solution and provide its security analysis.

Ethical Consideration: This work does not raise any ethical and legal concerns. The attacker and victim devices are part of a testbed setup established in our lab. We have especially purchased sim cards from two US operators to conduct our experiments. We did not use any other commercial sim card to launch the attack towards any other LTE subscriber. The purpose of this study is to strengthen LTE security, especially when LTE security mechanisms are considered to be the building blocks for $5 \mathrm{G}$ security (e.g. Cellular IoT security).

\section{BACKGROUND ON LTE AND KEY REINSTALLATION}

We provide background on LTE infrastructure, integrity and ciphering procedures in LTE, as well as on key reinstallation vulnerabilities.

\subsection{LTE network and its elements}

LTE device It provides LTE service to end user. The network operators assign the subscriber device a permanent identity called International Mobile Subscriber Identity (IMSI), and a number of temporary identities. One of these temporary identities is called Temporary Mobile Subscriber Identity (TMSI), a temporary identification number that is used instead of the IMSI to ensure the privacy of the mobile subscriber. The other temporary identity is known as RNTI (Radio Network Temporary Identifier) that uniquely identifies an LTE subscriber over the radio interface. LTE device applies ciphering to its both control and data planes, whereas the integrity is applied to control-plane only.

LTE base station It acts as a radio interface between LTE subscriber and the core network. It provides radio resource management to its subscribers and encrypts user traffic over the air. Through RNTI, it discerns a particular user traffic from other subscribers over the air. The control-plane radio signaling messages between device and LTE base station are exchanged through Radio Resource Control (RRC) protocol. RRC is responsible of activating radio-plane security (through Security Mode Command procedure) and managing the radio resources (such as establishment, release, and radio configurations).

LTE core network It is also called Evolved Packet Core (EPC) which acts as a central entity and provides authentication, mobility management, and Internet connectivity to LTE subscribers. The control-plane signaling messages between device and LTE core network are exchanged through Non-Access Stratum (NAS) procedures. NAS procedures include device Authentication (that installs security key), Security Mode Command (that enables ciphering), Attach (registration), Detach (de-registration), Tracking Area Update (location update), and few others procedures. In this paper, we first exploit vulnerabilities in Authentication and Security Mode Command procedures, and then launch attacks towards Tracking Area Update (TAU) and Detach control-plane procedures.

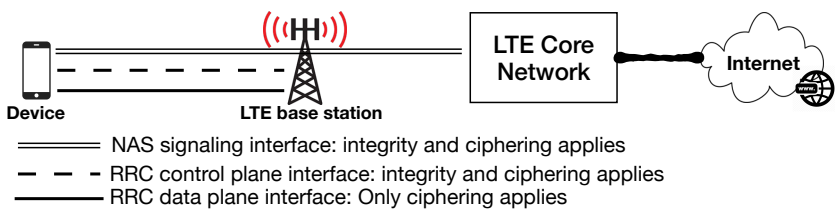

Figure 1: LTE background: an overview.

\subsection{Integrity and confidentiality procedures in LTE}

LTE employs integrity and confidentiality procedures which are applied at both device and network side. Figures 2(a) and 2(b) show integrity and ciphering procedures, respectively. LTE uses two separate algorithms for integrity and ciphering of messages. Both algorithms take a number of input parameters and output the Message Authentication Code (MAC), if integrity algorithm is used, or keystream block, if ciphering algorithm is used. As shown in Figure 2, the input parameters are 128-bit integrity/ciphering key named KEY, a 32-bit count named Count, a 5-bit bearer identity, i.e. Bearer, the 1-bit direction of the transmission i.e. Direction (0 for uplink, and 1 for downlink transmission). The integrity algorithm takes the message itself, i.e. Message, as input as well, and outputs MAC; whereas, the ciphering algorithm inputs the length of the keystream required i.e. Length, to generate the output keystream block. This Length parameter affects only the length of the keystream block, not the actual bits in it[7]. The keystream
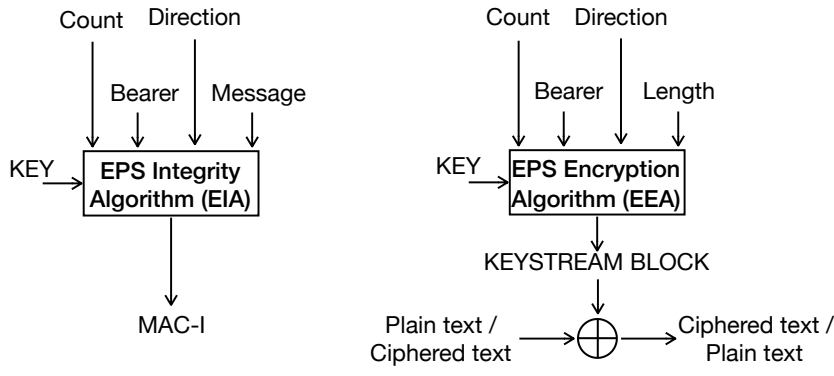

(a) Integrity protection

(b) Ciphering / unciphering

Figure 2: Integrity and ciphering procedures. 
block is xored with characters in the plaintext to produce the ciphertext at sender side. Xoring the ciphertext with same keystream block produces the plaintext at receiver.

\subsection{Key reinstallation attack in retrospect}

Stream ciphers, as discovered by Gilbert Vernam in 1917 [37], have been a popular method of encryption even today. In a stream cipher, the plaintext and the key are xored to produce the ciphertext. This cipher is never used again and known as one-time pad for encrypting plaintext message. To ensure that all ciphers do not occur more than once, the ciphering algorithm takes the nonce (we call count in this paper) as an input along with the key. Counts have the property that each value only occurs once within a given context. As long as the key is unchanged, the count must not repeat. Otherwise, it introduces the two-time pad problem [21] in which the adversary can get the encrypted plaintext without knowing the key and count values. The key reinstallation attacks can be defined as the attacks in which the adversary can willfully trick the victim to reuse the count values while keeping the key unchanged for encryption of the plaintext messages. It means stream cipher is reused for encryption, hence gives birth to two-time pads[21].

In wireless networks, David Wagner and his team have first shown stream cipher reuse attacks in WiFi[11], and WSN[16]. Following a number of papers $[8,10,40]$ afterwards, last year, Mathy Vanhoef and et al.[35] show that the key reuse attacks are still possible in modern WiFi systems. They attribute this problem to design or implementation flaws. In contrast, in this paper, we are first to show that LTE security is vulnerable to key reinstallation attacks. It was challenging because unlike WiFi and WSN, LTE has separate security keys and counts for control-plane and data-plane operations. Even within the control-plane, RRC and NAS messages use separate security keys and counts for their messages integrity and ciphering. Furthermore, LTE security also splits its counts into Uplink (UL) count and Downlink (DL) count values that make count reuse harder. Despite all these efforts by LTE standard to avoid key reuse, we have shown the key reinstallation attacks in LTE control plane.

\section{SYSTEM SETTINGS AND THREAT MODEL}

System settings The attacker controls LTE device (i.e. attacker device) that is associated with the same LTE network operator as that of victim subscriber. Both attacker and victim are located in an area where the network operator supports both 3G and 4G LTE services. The attacker knows the phone number of victim device, and can dial Circuit Switched Fall Back (CSFB) call towards victim device $^{1}$. The victim device can receive the call either through CSFB or Voice over LTE (VoLTE). Lastly, we consider both the attacker and victim devices are static during the attack period. That is, we do not evaluate the mobility scenarios.

Threat Model Similar to threat models as discussed in [14, 29, 35], our attacker has capability to act as a passive and an active attacker. Being a passive attacker, he can sniff radio channel with which the victim has associated. He can do so by sniffing Physical Downlink Shared Channel (PDSCH). PDSCH is used to transport

\footnotetext{
${ }^{1}$ The attacker selects CSFB option (which is voice call option over $3 \mathrm{G}$ ) in android/iOS phone call settings.
}

both broadcast system information for all devices and signaling payload for particular mobile devices. The attacker identifies different subscribers through their unique radio identity, C-RNTI.

Being an active attacker, he has capability to modify the contents of the messages (after decryption) that he has sniffed over the air. There exists a number of commercial LTE signal messages sniffers, such as WaveJudge[1], ThinkRF[5], and others that the attacker can use to sniff both broadcast and device specific signaling messages. Contrary to attack models discussed in $[29,35]$, our attack model is more practical in which the attacker does not need to act as Manin-the-Middle (MitM) to forward modified messages towards the network. To impersonate the victim device, if required, the attacker spoofs victim's C-RNTI and TMSI values when he creates his own RRC and NAS messages, and sends his signaling messages to LTE base station. The spoofing is essential to trick LTE base station to use victim context (not the attacker's context) while forwarding message to core network.

In order to ensure that failure of certain signaling messages result in reseting the count values, the attacker has capability to block UL (from device to network) signaling messages. He achieves this by jamming UL signaling. There are a number of techniques[19, 20, 23] to jam the signaling messages. We consider Asynchronous Off-Tone Jamming (AOTJ) approach to jam only UL signaling messages between victim device and the network. The core idea of jamming is to introduce the inter-channel interference (ICI) among orthogonal OFDM subchannels. The interference brings loss of subchannel orthogonality, and as a result the network cannot recover the original OFDM data symbols over its subchannels which are spectrally overlapping. In our AOTJ technique, the jammer is off-tone or not synchronous with the target signal. It transmits asynchronous offtones which are not perfectly periodic or have an offset at the sampling frequencies that brings ICI at the receiver.

Evaluation of attacks We evaluated our attacks in terms of their feasibility and practicality over real operational LTE networks. We use Google Pixel 2 as an attacker device, and Google Pixel 1 as victim device. We consider two U.S LTE operators, i.e. AT\&T (OPI) and T-mobile (OPII) to run our experiments. The attacker and victim devices use AT\&T and T-mobile pre-paid sim cards to register with these two network operators. We use LTE signaling messages analyzer, MobileInsight, to capture LTE signaling messages at both attacker and victim devices. We run total of 200 experiments on each network operator to assess the practicality of attack for each attack step. To evaluate the practicality of the attacks, we use lowcost commodity SDR hardware (HACKRF One) of the value of \$299 to jam LTE signaling messages. HACKRF One has capability to block UL and DL LTE signaling messages by generating ICI signals towards LTE frequency band. To calibrate start and stop of jamming with respect to LTE signaling messages, we use QXDM[3] which is a real time LTE signaling messages sniffing/capturing tool from Qualcomm.

\section{OVERVIEW ON ATTACKS AND THEIR ROOT CAUSES}

The attacker can launch two types of key reinstallation attacks by exploiting signaling vulnerabilities. In the first type, he hijacks the location update procedure of the victim device. Consequently, 
the network cannot reach the victim for the incoming voice calls and data packets destined to the device. In the second type, the attacker may incur LTE service outage at the victim device by deregistering the device from the network. In our experiments, we demonstrate the feasibility of these attacks over real LTE carrier networks and their practicality in our testbed. There are two root causes for the attacks. The first root cause is that the LTE controlplane procedure is vulnerable to key reinstallation attacks. Such attacks arise when the count reset procedure (i.e. LTE Security Mode Command procedure) is allowed to re-execute many times after the completion of key installation procedure (i.e. LTE NAS Authentication procedure). The second root cause is that certain control-plane messages are partially accepted even though they fail the integrity check. These messages are finally accepted when the network re-authenticates the device. The network does not request the device to re-send the message that has failed the integrity check.

\section{ATTACKING LTE CONTROL PLANE}

Overview We demonstrate the feasibility and practicality of key reinstallation attacks in LTE control-plane. The adversary adopts the fact into his advantage that on inter-system switch from LTE $\rightarrow 3 \mathrm{G} \rightarrow \mathrm{LTE}$, location update procedure is triggered that installs the key and resets the count values. The attacker silently ${ }^{2}$ brings an inter-system switch at victim device through CSFB procedure. He lets the device to complete the key installation procedure, but strategically blocks the victim device UL signaling messages to bring count reset procedure failure. The network re-initiates the failed procedure that resets the count values at device again. This results into keystream block reuse for those signaling messages that the victim device sends after resetting the count values. The attacker stops jamming, encrypts his spoofed message by using victim's keystream block ${ }^{3}$, and dispatches it to the network without being MitM. The network receives two messages, the one originated from the device and the other from the attacker. The network executes the latest received message, according to 3GPP standard[6], and discards the message received earlier. This makes our attack realistic as the attacker message and the victim messages are not racing with each other. Because the message was modified by the attacker, it fails the integrity check at the network. However, instead of dropping the packet, the network re-authenticates the victim device and accepts the received spoofed message.

\subsection{LTE Location Hijacking Attack}

5.1.1 Feasibly analysis from LTE standard. Following we discuss two vulnerabilities that we exploit in attacking LTE confidentiality and integrity protocols.

1.1 LTE Integrity and confidentiality are enforced through two disjoint procedures LTE security is enforced through two separate procedures. In the first procedure, the LTE core network invokes mutual authentication procedure, i.e. AKA procedure, with the subscriber device. In LTE AKA procedure, as shown in Figure 3 (upper rectangular part), the core network element sends an Authentication Request message to the device.

\footnotetext{
${ }^{2}$ Attacker terminates the call before the victim device starts ringing, hence making it silent inter-system switch.

${ }^{3}$ Attacker gets the keystream block by xoring location update message with the encrypted message.
}

The device authenticates the LTE core network element, installs the key and sends the Authentication Response message to network. LTE core network verifies the response message and installs the key at its end. After authentication procedure, the network triggers NAS Security Mode Command (SMC) procedure. The network sends SMC message to device, as shown in Figure 3 (lower rectangular part) that includes NAS security algorithms to derive integrity and ciphering keys ${ }^{4}$, as well as NAS-MAC (NAS Message Authentication Code). As the device does not know the selected encryption algorithm yet, this message is integrity protected only but not ciphered. On receiving the SMC message the device resets the pair of count (one for UL and one for DL transmission) values to zero after NAS-MAC verification for integrity protection. The LTE security specification (3GPP TS 33.401[7]) states:

"Only after EPS AKA the NAS security mode command message shall reset NAS uplink and downlink COUNT values. Both the NAS security mode command and NAS security mode complete messages are protected based on reset COUNT values (zero)."

Thereafter, the device generates NAS Security Mode Complete message to network which is both ciphered and integrity protected. The network successfully verifies the integrity of the received NAS Security Mode Complete message and resets the counts. Now the NAS security setup procedure is said to be completed.

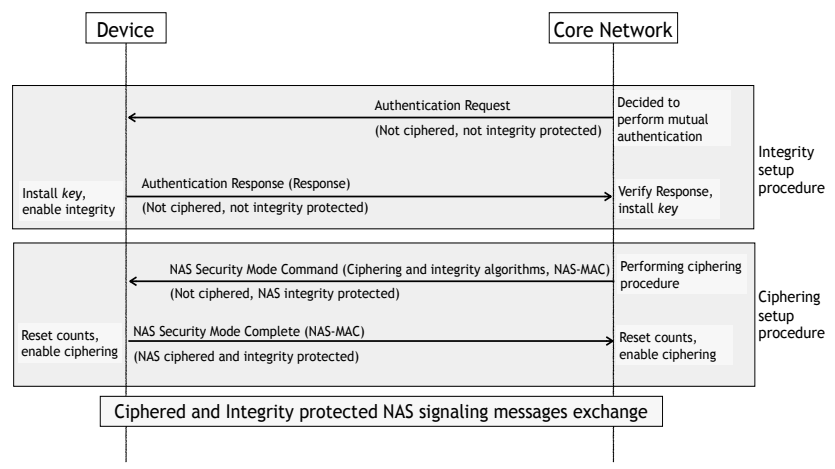

Figure 3: Authentication procedure installs security key and enables integrity protection at the device and the network. The NAS Security Mode Command procedure activates ciphering at the device and the network sides after successful completion of the authentication procedure.

Now it is easy to see the vulnerability in which the attacker exploits the fact that the device resets the count values after installing the key. The attacker can block the transmission of NAS Security Mode Complete message and lets the network to re-initiate the SMC procedure; causing the device to reset the counts again. In this way, the signaling messages sent by device between subsequent SMC procedures use same keystream block for their encryption. Vulnerability 1: Failure of SMC procedure does not renew the security key.

1.2 Network accepts certain NAS messages that fail the integrity check It is understandable that a number of NAS signaling messages can be exchanged between device and the network before the activation of NAS security. These signaling messages include Attach Request, Authentication Request/Response/Failure, Security Mode Reject, Identity Response, and few others. However, there are a number of other messages (that include TAU Request and Detach

${ }^{4}$ For simplicity, in this paper we name all types of keys (i.e. integrity, ciphering) as key. 
Request/Accept messages) that are "conditionally" accepted when they fail the integrity check.

LTE NAS specification (3GPP TS 24.301[6]) states:

"These messages are processed by the MME even when the MAC that fails the integrity check or cannot be verified, as in certain situations they can be sent by the UE protected with an EPS security context that is no longer available in the network."

However, LTE core network re-authenticates the device before finally accepting the message. As stated in LTE NAS specification (3GPP TS 24.301[6]):

"If a TRACKING AREA UPDATE REQUEST message fails the integrity check, the MME shall initiate a security mode control procedure to take a new mapped EPS security context into use."

Such a 3GPP standard approach is vulnerable in which the network accepts the spoofed message, failing the integrity check, after re-authenticating the device.

Vulnerability 2: The network re-authenticates the device instead of rejecting certain messages failing the integrity check.

5.1.2 Detail attack procedure. We describe step by step attack procedure as follow.

Pre-condition Before launching the attack, the attacker needs to know the TMSI of the victim subscriber for identification purpose. The attacker gets the TMSI through broadcast paging message addressing the victim device. He can easily generate the paging message for victim device by simply calling the victim. If the victim phone is in idle state, the core network sends a paging broadcast message that includes victim's temporary identity (TMSI). On receiving the paging message, the victim device switches to connected state and prepares to receive its call. Because the paging is a broadcast message within the tracking area, the attacker device also receives the paging message[14]. By repeating this procedure, the attacker can ensure that the TMSI maps to the victim device (subscriber's phone number).

Experiment results: A clever attacker would hang-up the call before the victim device starts ringing. To assess the practicality of hanging-up the call so that the victim device does not start ringing, we run several experiments. We record the signaling messages and the time between call initialization and call ringing events. In our experiments, both caller and callee phones are time synchronized through which we accurately correlate the events between two phones. In total we collected 200 logs with 2 major US operators. We consider the cases when the victim device receives the call through CSFB, and VoLTE. The attacker always makes a CSFB call (by turning off VoLTE option at its phone).

After initiating the call, the attacker must wait for paging message to be delivered to victim device before hanging-up the call. We can see from Table 1 that it takes around 3.5 seconds and 4.6 seconds (on average) for paging message to be received at victim device for OPI and OPII, respectively. The attacker can terminate the call afterwards where he has the error margin of 3.3 seconds and 5.3 seconds (on average) to hang-up the call so that victim device does not ring for OPI and OPII, respectively. Table 1 also shows the results when the victim device receives the call through VoLTE instead of CSFB.

There is a possibility that the call from the attacker does not trigger any paging message towards the victim device. This is the
Table 1: Silently getting victim TMSI: The time margin the attacker has to hang-up the call by making sure that (1) paging message is broadcasted towards victim, and (2) victim device does not ring.

\begin{tabular}{|l|l|l|l|l|l|l|l|l|}
\hline \multicolumn{1}{|c|}{ Victim receives call through CSFB } \\
\hline Operator & \multicolumn{1}{|c|}{ Call init to paging msg } & \multicolumn{2}{|l|}{ Paging msg to call ringing event } \\
\hline & Min & Max & Avg & STD & Min & Max & Avg & STD \\
\hline OPI & $3.2 \mathrm{~s}$ & $6.1 \mathrm{~s}$ & $4.6 \mathrm{~s}$ & $0.5 \mathrm{~s}$ & $2.4 \mathrm{~s}$ & $4.4 \mathrm{~s}$ & $3.3 \mathrm{~s}$ & $0.4 \mathrm{~s}$ \\
\hline OPII & $2.5 \mathrm{~s}$ & $4.8 \mathrm{~s}$ & $3.5 \mathrm{~s}$ & $0.6 \mathrm{~s}$ & $3.5 \mathrm{~s}$ & $6.6 \mathrm{~s}$ & $5.3 \mathrm{~s}$ & $0.9 \mathrm{~s}$ \\
\hline \hline \multicolumn{8}{|c|}{ Victim receives call through VoLTE } \\
\hline Operator & Call init to paging msg & Paging msg to call ringing event \\
\hline \multicolumn{10}{|c|}{ Min } & Max & Avg & STD & Min & Max & Avg & STD \\
\hline OPI & $2.3 \mathrm{~s}$ & $4.4 \mathrm{~s}$ & $3.3 \mathrm{~s}$ & $0.5 \mathrm{~s}$ & $0.7 \mathrm{~s}$ & $2.0 \mathrm{~s}$ & $1.3 \mathrm{~s}$ & $0.3 \mathrm{~s}$ \\
\hline OPII & $2.2 \mathrm{~s}$ & $4.6 \mathrm{~s}$ & $3.3 \mathrm{~s}$ & $0.6 \mathrm{~s}$ & $1.6 \mathrm{~s}$ & $2.6 \mathrm{~s}$ & $2.2 \mathrm{~s}$ & $0.3 \mathrm{~s}$ \\
\hline
\end{tabular}

case when the victim device is in connected state. From Table 1, we can see that the attacker can easily determine whether the victim device is in idle or connected state. He first waits from call init to paging message triggering time. If he does not sniff the broadcast paging message during this period then he assumes that the victim device is in connected state. The attacker then backs-off for tens of seconds (the device's default inactivity timer - time to transition from connected to idle state - is 10s) and retries the call.

We now discuss our attack procedure in 3 main steps as shown in Figure 4.

(1) Triggering key update through inter-system switch The attacker's goal is to install fresh key and reset count values at victim device. To achieve this, he dials a phone call towards victim to get CSFB call connection established with victim device and then hangs-up the call. The CSFB call forces victim device to perform inter-system switch (from LTE to $3 \mathrm{G} / 2 \mathrm{G}$ ). Once the attacker hangsup the call, the victim device moves back to LTE (from 2G/3G) and performs random-access channel (RACH) procedure to synchronize with LTE base station. Through RACH procedure, the device receives a temporary radio identity (C-RNTI) mapped with its TMSI from the base station. The attacker sniffs RACH messages to associate victim subscriber's TMSI with its C-RNTI. After RACH procedure, the device setups its radio connection and sends unciphered TAU Request message as initial NAS message. The device also starts timer T3430 (default value of 15s) to retransmit the TAU Request message if timeout occurs. On receiving the TAU Request message, the network performs the Authentication procedure through which both victim device and the network authenticate each other and install the key.

Experiment results: We run more than 200 experiments to assess the practicality of the attack. At first, we assess how successfully an attacker can trigger inter-system switch by dialing a phone call. We find that there are two cases: (1) either victim device or the network does not supports VoLTE feature; or (2) both the victim device and its associated network support VoLTE. In case of (1) the victim device automatically switches to circuit switched network, i.e. $2 \mathrm{G}$ or $3 \mathrm{G}$, to receive the call. However, in case of (2) the victim device does not performs automatic inter-system switch, and the attacker needs to enforce it. From our experiments, we find that if the VoLTE call is blocked at device for 5 seconds then the LTE modem chipset (Qualcomm LTE modem) aborts VoLTE call in favor of making the call through CSFB. This feature has also been reported in several other studies [33, 34]. Now, the attacker strategy is to temporarily block (through UL jamming) the signaling messages between victim device and its network. But the question arises (i) when to start jamming after dialing the call?; (ii) how long the attacker can delay 
in starting jamming because in practice it is hard to start jamming at a precise time?; and (iii) when the attacker should hang-up the call after stopping jamming so that the victim device does not ring? For (i), table 2 shows error margin with min, max and average values of $2.2 \mathrm{~s}, 4.3 \mathrm{~s}, 3.3 \mathrm{~s}$ with standard deviation of $0.5 \mathrm{~s}$ for attacker to start UL jamming. That is, the time he has from initiating the call to sniffing the paging message (voice call indication for victim device in idle state). Once the attacker has decided to start UL jamming, he has an error margin of $0.4 \mathrm{~s}$ (on average) with standard deviation of $40 \mathrm{~ms}$ to start jamming as shown in Table 2. This is the time in which victim device establishes the VoLTE call connection with the network, answering question point (ii). The jamming lasts for $5 \mathrm{~s}$ that induces victim device to perform CSFB procedure to establish voice call connection over $3 \mathrm{G} / 2 \mathrm{G}$ network instead of LTE. The attacker hangs-up the call before the victim device rings (i.e. within 3.3 seconds - refer to Table 1 Paging to Call ringing time after stopping the jamming) which addresses our question point (iii). On hanging-up the call, the device switches back to LTE and performs RACH procedure that facilitates attacker to map TMSI with C-RNTI. The attacker has on average $45 \mathrm{~ms}$ (10ms of STD) to capture RACH Response and/or RRC Connection Request message to successfully establish mapping, as shown in Figure 5(a).

Table 2: Forcing victim to establish CSFB call connection instead of VoLTE: The error margin in terms of time the attacker has to start UL jamming so that the victim device fails to establish the VoLTE call connection. As a result, the victim phone receives call through CSFB procedure.

\begin{tabular}{|l|l|l|l|l|l|l|l|l|}
\hline Operator & \multicolumn{4}{|c|}{ Call init to call indication } & \multicolumn{3}{|c|}{ Paging to VoLTE connection } \\
\hline & Min & Max & Avg & STD & Min & Max & Avg & STD \\
\hline OPI & $2.2 \mathrm{~s}$ & $4.3 \mathrm{~s}$ & $3.3 \mathrm{~s}$ & $0.4 \mathrm{~s}$ & $0.3 \mathrm{~s}$ & $0.6 \mathrm{~s}$ & $0.4 \mathrm{~s}$ & $0.04 \mathrm{~s}$ \\
\hline OPII & $2.2 \mathrm{~s}$ & $4.6 \mathrm{~s}$ & $3.3 \mathrm{~s}$ & $0.6 \mathrm{~s}$ & $0.4 \mathrm{~s}$ & $0.7 \mathrm{~s}$ & $0.5 \mathrm{~s}$ & $0.04 \mathrm{~s}$ \\
\hline
\end{tabular}

(2) Administrating key reinstallation attack through onetime jamming After the authentication procedure, the core network activates the Security Mode procedure by sending integrity protected SMC message to the device and sets the message retry timer T3460 (default value of 6s). The attacker who is sniffing the radio traffic finds the SMC message matching the victim's C-RNTI and starts UL jamming. The attacker has the error margin of 2 messages in starting UL jamming (i.e. either after sniffing Authentication Response message, or Security Mode Command message). On receiving the SMC message from the network, the device verifies message integrity, resets counts (vulnerability 1 in Section 5.1.1), and sends Security Mode Complete message to the network. Because this UL message from device is blocked over the air, the network does not receive this response message and its timer T3460 expires. The network re-sends SMC message to victim device by resetting the timer T3460. The victim subscriber resets its UL/DL transmission count values and sends the Security Mode Complete message which is blocked as well by the attacker. Similarly, the third response to network initiated Security Mode procedure is also blocked. Meanwhile, the TAU timer T3430 at victim device times out. At this point, the device has already enabled ciphering (as it has sent out Security Mode Complete messages thrice). The victim subscriber prepares new TAU Request message and applies ciphering and integrity protection. It sends out the TAU request message which the attacker sniffs and stores it at his end. We call this message $T A U_{1}$, that is TAU Request message 1 which is encrypted with keystream block ${ }^{5}$. Note that the attacker can recover the TAU message as he himself is jamming resilient. This is because he knows his off tone jamming signals and can cancel interference added to jam the signals[19, 22, 38, 39]. However, the TAU message is non-decodable at the network side due to unknown interference. When the Security Mode procedure fails for the fourth time, the attacker stops UL jamming. As a result, the Security Mode procedure succeeds on its fifth try where the network resets counts and enables ciphering its end. From this point onward, the network only accepts messages which are both integrity protected and ciphered. Experiment results: In order to make the attack practical, the attacker has to ensure that he (i) identifies the victim over the radio before starting UL jamming, and (ii) starts UL jamming before Security Mode Command complete receives at the network. For (i), he has an error margin of $380 \mathrm{~ms}$ on average (with STD of $20 \mathrm{~ms}$ ) to identify the victim device through PDSCH. This is the time between RRC Connection request and Security Mode Command messages, as shown in Figure 5(b). For (ii), the attacker has on average $48 \mathrm{~ms}$ (with 5ms STD) to start UL jamming (after Authentication Response message but before Security Mode Complete message), as shown in Figure 5(c).

We also perform more than 200 lab experiments to assess the success probability of starting jamming within the specific time interval (i.e. $48 \mathrm{~ms}$ ). For this, we first use Qualcomm real time packet sniffing tool QXDM[3] to calibrate the time between performing inter-system switch and starting UL jamming. We modify the HACRF One source code to make jamming effective, and achieve UL and DL frequency jamming within $1 \mathrm{~ms}$ after its initialization. We face two challenges in jamming specific LTE signaling message(s). From our experiments, we find that when we jam signals for more than 6 seconds the device internally triggers radio link failure, and if we continue jamming then the device switches to $3 \mathrm{G}$ network. To address this challenge, we systematically switch on and off jamming in an interval of $2.5 \mathrm{~s}$ such that desired signaling messages remain blocked when they are re-transmitted on their time-out. The other challenge we face was regarding jamming UL signaling messages. We find that the device increases its UL transmit power (as high as $25 \mathrm{dBm}$ whereas our HACKRF One max UL transmit power is $15 \mathrm{dBm}$ ) that renders UL jamming through low cost SDR device ineffective. To address this challenge, we perform DL jamming instead and block the TAU Accept message reaching towards the device. As the TAU procedure does not succeed after all, the network responds to retransmitted TAU request messages (as well as the spoofed message to be discussed in the next step below) even if it has received TAU request message earlier. Hence, we can successfully execute our attack step in practice.

On practicality of jamming: We briefly discuss that our jamming works even if the attacker lacks LTE dedicated channel sniffing capability. We can always start jamming at desired signaling message with high probability. To evaluate this, we use three different methods, as discussed below:

Straw-man approach: The attacker makes a CSFB call towards victim, hangs-up the call as soon as the victim subscriber receives

${ }^{5}$ Obviously, this message is also integrity protected, but we are interested to break the ciphering only to carry out our attack 


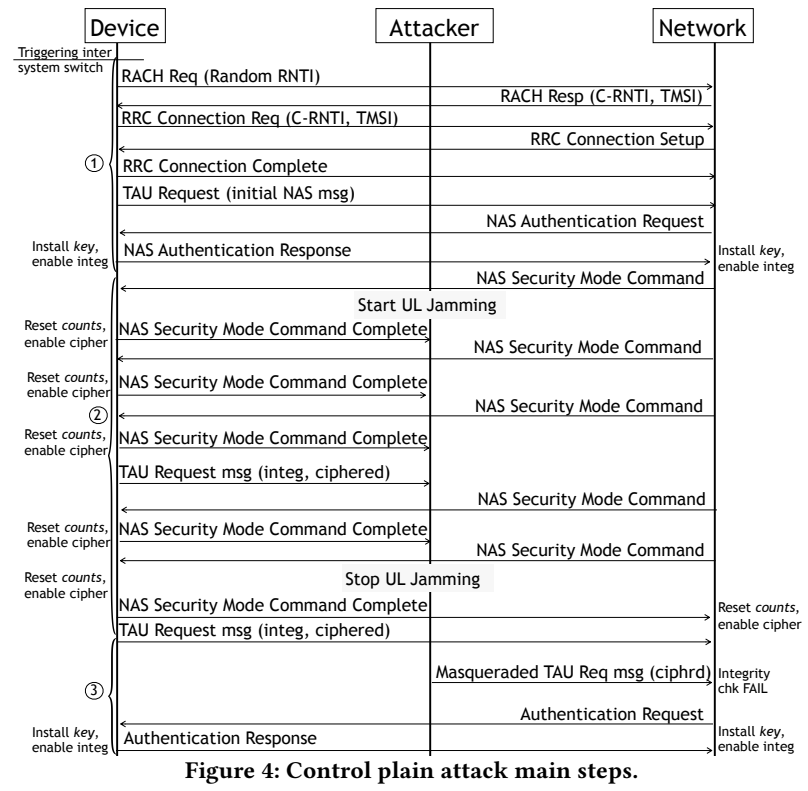

paging message, and starts jamming after waiting for $450 \mathrm{~ms}$ (calculated according to Figure 5). We see that in this case the success probability of jamming is just $21 \%$. This is mainly because the attacker hangs-up call while victim device was in the middle of call establishment procedure. This triggers location update procedure in $3 \mathrm{G}$ and the device does not release the connection towards LTE network.

Measured approach: To address the problem of straw-man approach, we let the control-plane call establishment procedure to be completed before hanging-up the call. The attacker lets the call establishment procedure to be completed before it hangs-up the call (just before call ringing). Hanging-up the call at this time triggers RRC connection release towards LTE network and the victim device immediately switches back to LTE network. The attacker starts the jamming after waiting for $450 \mathrm{~ms}$ and gets the desired message blocked with the accuracy of $58 \%$. The accuracy is halfed due to variable time of inter-system switch (i.e. how quickly LTE cell is selected).

Adaptive approach: Instead of calculating the jamming start time from call release event, we improve our results by sniffing the LTE broadcast $\mathrm{RACH}$ packet before making the jamming decision. Our results improve the jamming accuracy to $78 \%$ because in reality we cannot $100 \%$ predict when control-signaling message will arrive in future.

In summary, we show that the jamming at the desired occasion can be achieved with the accuracy of roughly $80 \%$ even if the attacker does not sniff LTE dedicated channel.

(3) Spoofing location update messages through keystream block reuse Because the attacker has stopped jamming in step (2), the device initiated TAU request (on expiry of TAU timer T3430), we call it $T A U_{2}$, arrives at the network ${ }^{6}$. The attacker sniffs this TAU request message as well and retrieves the keystream block by

${ }^{6}$ Careful reader should note that T3430 times out earlier than TAU Accept timer T3450 (default value 6s) at the network therefore network does not send TAU Accept message on receiving Security Mode Complete message
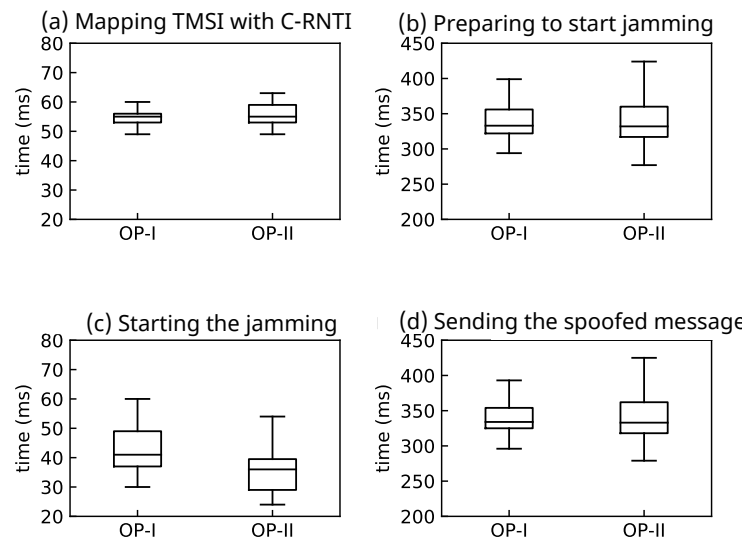

Figure 5: Error margin (min, max, avg, and std) for different experiments. Time between (a) RACH Request to RRC Connection Request messages; (b) RRC Connection Request to Security Mode Command messages (c) Authentication Response to Security Mode Complete messages; (d) Security Mode Complete (5th try) to TAU Request (3rd try).

xoring either the contents of $T A U_{1}$ or $T A U_{2}{ }^{7}$. Recall that, he already gets hold of TAU request message (as initial NAS message) sent in plain text in step (1). Once he retrieves keystream block from the ciphered text, he encrypts his spoofed TAU request message that includes wrong device location identity by xoring the retrieved keystream block. He replaces his C-RNTI with victim's one ${ }^{8}$ and immediately sends the spoofed message to the network. The network receives the spoofed TAU request message while it was waiting for TAU Complete message from device (as the network sends TAU Accept message after receiving $T A U_{2}$ ). According to LTE 3GPP standard, the network aborts previously received TAU message and processes the newly arrived message with different location identity (i.e. location information element). It has been stated in LTE NAS specification (3GPP TS 24.301[6]):

"If one or more of the information elements in the TRACKING AREA UPDATE REQUEST message differ from the ones received within the previous TRACKING AREA UPDATE REQUEST message, the previously initiated tracking area updating procedure shall be aborted if the TRACKING AREA UPDATE COMPLETE message has not been received."

The network decrypts the attacker originated TAU message and checks the integrity of the message. As the message contents were modified by the attacker, the TAU request fails the integrity check. The network finds that this is a special NAS message (4.4.4.3 Integrity checking of NAS signaling messages in the MME [6])) and it should be processed when the device fails the integrity check (vulnerability 2 in Section 5.1.1). However, before accepting the message, the network successfully authenticates the victim device (by initiating the Authentication procedure), and sends TAU Accept message to the victim device. The victim devices replies with TAU Complete message to network that registers the spoofed device location identity in its database.

${ }^{7}$ Careful readers will argue that why the attacker needs to wait for second retransmitted TAU when he can create spoofed message at step (2). We do so to avoid the victim device transitioning back to registered state from TAU init state when the TAU timer T3430 expires while the spoofed TAU is being processed at the network. That can invalidate our attack in which the device initiated TAU rectifies the location identity ${ }^{8} \mathrm{C}$-RNTI spoofing is necessary so that LTE base station forwards the attacker's spoofed message towards victim's S1AP connection. 
Experiment results: To make this step successful, the network must receive the attacker's spoofed message before the TAU Complete message arrives from the victim device. This is the time between receiving Security Mode Complete and TAU Complete messages (a device response to TAU Accept message of $T A U_{2}$ ). From Figure 5(d), we can see that the attacker has on average $370 \mathrm{msec}$ (15msec STD) to prepare and send its spoofed message to the network. For validating the impact of spoofed message, we modify the non-volatile memory of the LTE modem and used Qualcomm's service-programmer tool (QPST Service Programmer)[2], and ATcommand tool (TeraTerm)[4] to send the spoofed message.

5.1.3 Attack damage. The consequence of our attacker is that the network updates the victim device location to erroneous tracking area. When the victim device enters in the idle state, it releases the RRC connection. The device relies upon the paging message from the network for the notification of its data packets during its idle state (e.g. if someone sends a text message, or voice call to victim). Because the attacker has registered the victim device on wrong location by hijacking TAU procedure, the victim device does not receive the paging message. Hence, the victim device remains unreachable for its incoming voice and data traffic.

Constraints: To realize the attack, the device must transition to idle state after performing the TAU procedure. The maximum time the victim device remains under attack is the time until it performs periodic TAU procedure (default value of 54 minutes). Note that, other LTE procedures such as Service Request procedure or VoLTE call establishment do not have any impact on our attack (i.e. they do not shorten the attack time).

Extending the attack period: The attacker can easily re-launch the attack to keep the victim device under attack even if the device updates its location through periodic TAU procedure, establishing a CSFB call, or even rebooting. After launching the attack for the first time, the attacker periodically pages the victim device by initiating a call towards him. If attacker's call generates the paging message, it means the victim subscriber has recovered from the attack. The attacker then re-launches the attack by following steps (1) to (3), and keeps the victim subscriber under attack.

\subsection{Designing LTE Service Outage Attack}

We extend our location hijacking attack to bring more serious attack. In this variant of the attack, the attacker sends Detach Request message (with cause power off) instead of sending the spoofed TAU request message at step (2) to the network. There are two scenarios that occur at the network. First, the network receives the device de-registration request in the middle of ongoing TAU procedure (i.e. the network is waiting for TAU Complete message from the device). Second, the detach request being sent by the attacker is bound to fail the integrity check at the network. The 3GPP standard explicitly discusses both these cases in LTE NAS standard[6]. The first case is defined as abnormal case for TAU procedure that requires the network to abort the TAU procedure and to process the Detach Request message from the device. It has been stated in [6]:

"If the device receives a DETACH REQUEST message before the tracking area updating procedure has been completed, the tracking area updating procedure shall be aborted and the detach procedure shall be progressed."
While progressing the detach request message, the network finds the message has failed the integrity check. This is our second scenario and the 3GPP standard requires the Detach Request message (with cause power off) must be processed even of the message fails the integrity check (i.e. our vulnerability 2 in Section 5.1.1). LTE NAS specification states [6]:

"The procedure is completed when the network receives the DETACH REQUEST message. On reception of a DETACH REQUEST message indicating "switch off", the MME shall delete the current EPS security context."

We must point out that this special case only applies to Detach Request with reason power-off, otherwise, the network proceeds with the tracking area updating procedure first before progressing the detach procedure.

No LTE service When the network receives Detach Request message with cause power off, it re-authenticates the victim device first and then releases the device connection by deleting device sessions and freeing its IP address. The device (being unaware of its network registration has terminated) sends Service Request message (when it has some data to send or call to initiate). On receiving the Service Request message from the victim device, the core network rejects the request with error cause \#43 (Invalid EPS bearer identity). On receiving the Service Reject message with error cause \#43, the device enters into deregistered state, according to 3GPP NAS specification[6] that states:

"The UE shall abort any ongoing ESM procedure related to the received EPS bearer identity, stop any related timer, and deactivate the corresponding EPS bearer context locally (without peer to peer signalling between the UE and the MME)."

Now the victim needs to manually register the device with network (by rebooting device or by turning on/off the device airplane mode), otherwise LTE service remains unavailable.

\section{DISCUSSION}

We put forward remedies to the key reinstallation attacks and discuss security analysis of the proposed remedies.

\subsection{Proposed Remedies}

In this section, we suggest our LTE standard compliant remedies to address the discussed vulnerabilities and attacks.

1. Bounding key installation and count reset procedures: One of the root causes of control-plane attacks is the disjoint execution of key installation and count reset procedures. To address this, we bound LTE NAS Authentication (that installs the key), and NAS Security Mode Command (that resets count) procedures. That is, we perform LTE Authentication procedure whenever Security Mode Command procedure fails (making security procedures atomic). In LTE Authentication procedure, the network sends Authentication Request message by starting timer T3460 (default value of $6 \mathrm{~s}$ ). The timer is stopped when the network receives Authentication Response message from the device. In our solution, we stop T3460 when the network receives Security Mode Complete message from the device instead of stopping at Authentication Response. It means, the Authentication procedure fails if the device Security Mode Command procedure fails; hence bounding these two procedures. Our approach addresses vulnerability 1 where we 
make key installation and count reset procedures atomic; i.e. either both succeed or none .

2. Enforcing integrity protection for all signaling messages once security has been established: The other root cause of control-plane attacks is that certain messages (i.e. TAU and Detach Request) are partially accepted even if their integrity check fails. Although, the network authenticate the device afterwards but does not validate whether the received signaling message was indeed originated by the authenticated device or not. We mitigate this vulnerability by enabling the device to not accept any signaling messages failing the integrity check if the security association has already been established. Instead the network rejects the message and re-authenticates the device. We should point out that present 3GPP specifications generate integrity failure message response for selected signaling procedures. To provide the integrity check failure feedback for all types of signaling messages, we propose that the network should reject the signaling message with error cause \# 9 (UE identity cannot be derived by the network). On receiving this error message, the device re-registers with the network after executing both authentication and security mode procedures. Our standard compliant solution may arguable delay LTE service for a couple of seconds, but it enforces LTE security at all times; hence mitigating vulnerability 2 in which message failing the integrity is always re-executed.

\subsection{Security Analysis through Prototyping}

We provide the security analysis of our proposal by developing a proof of concept prototype without creating interaction between victim and attacker. We use AT commands to take certain actions emulating the network enforcing above principles to mitigate vulnerabilities 1 to 3. Although there exists hundreds of AT commands, only few have privilege to execute over commercial handsets. We create our prototype by using those AT commands which our program can execute over commercial phones (such as Google Pixel or Samsung Glaxy devices).

In our experiment, we check whether the subscriber device is under jamming attack or not. If the signals are jammed to launch key reinstallation attacks by resetting counts, we re-activate LTE bearers that re-establish the security by renewing key. When the device makes a voice call through CSFB, our program checks for LTE registration by running "at+creg?" and "at+cgdcont?" commands. "at+creg?" tells whether the device is PLMN registered and if true then whether it is registered with LTE network or not. The "at+cgdcont?" outputs the IP and APN name that explains with which cellular radio access technology the device has camped-on. For example, fast.tmobile.com tells the device is registered with LTE APN over T-mobile carrier network. Thereafter, our program sends "at+cgdata= "PPP", 1" command to establish the data connection with the network. If the data request is not entertained, the device AT command returns error. It means the device data connection request has failed due to jamming. On receiving the error message, our program waits for 2 seconds before running "at+cgdata" command again. If the error persists then our approach is to renew the key by re-activating LTE service. We run " $a t+c o p s=2$ " immediately followed by " $a t+c o p s=0,1$ " to force the device to reselect LTE network and perform re-authentication procedure.

\section{RELATED WORK}

We compare our related work with (1) key reinstallation attacks, and (2) LTE security.

Key reinstallation attacks: [28] has discussed key reinstallation attack on voice over LTE and fail to decrypt LTE signaling messages. [28] has shown the feasibility of the attack arising from LTE bearer assignment with IMS. In contrast, we have demonstrated the key reinstallation on control plane. Closest to our work is key reinstallation attack in WiFi[11, 32, 35]. Mathy and et al. [35] has recently shown a variant of key reinstallation attack in WiFi signaling plane. Their work exposes design and implementation issues in WiFi security protocols that reinstall an already-in-use key. [11] discusses passive and active attacks due to keystream reuses in WEP. [32] shows key recovery attack on WEP. In contrast, our work although in the similar direction is different than all above works. We show key reinstallation attacks in LTE control plane, even though LTE never reuses the same key (all keys are chained in forward direction), employs separate keys and counters for encryption and integrity protection.

Other works related to key reinstallation attacks are count reset due to power failure[40]; use of static counter due to implementation bugs[10]; faulty state machine transitions leading to count resets[9, 12]; count resets through routing protocols[8]; and side channel attacks on $\mathrm{CBC}$ mode with a block cipher[36]. Contrary to these works our work study LTE design flaws that resets counter values welcoming key-reinstallation attacks. Our attacks are neither implementation bugs nor brought due to careless design choices. We show that seemingly working security protocols have security loopholes when certain signaling messages are re-transmitted.

LTE security: A number of other works discuss LTE security issues. [14, 27, 29] conduct LTE protocol vulnerability analysis and show real impacts on LTE subscribers. LTEInspector[14] tool cannot detect key reinstallation vulnerabilities mainly because retransmission of lost message is a valid behavior of every protocol and it is hard to automate impact of these lost messages over LTE security. LTEInspector[14] findings on detach request with IMSI are different than what we disclosed in this paper. [27] discusses LTE inter-protocol vulnerabilities in which the adversary can spoof LTE messages. Their attacks were not practical because the network rejects the attacker originated message (as C-RNTI and TMSI do not match). [29] shows the MitM attacks to exploit LTE layer two vulnerabilities. Attacks discussed in [29] are mostly passive in nature that map device activities and perform website fingerprinting. In contrast, in this paper, we present novel attacks in LTE and make them practical through key stream reuse.

Other works such as [13] shows that LTE temporary identity can disclose subscriber location. [30] unveils that signaling information can be leveraged to infer user privacy. [26] shows that current cellular infrastructures exhibit security loopholes due to their NAT/firewall settings. [24, 25] study insecurity in mobile data charging. $[17,18]$ discuss how a subscriber can inject control-plane traffic into user-plane. Different to all above works, we do not discuss vulnerabilities due to misconfigurations or implementation bugs. 


\section{CONCLUSION}

This is the first work to best of our knowledge that shows key reinstallation attacks in LTE control plane. The re-transmission of certain signaling messages resets the counter values multiple times that lead to reuse of key stream block for ciphering of plain text messages. In consequence, the attacker can launch attacks on LTE control plane where he can hijack LTE location update procedure, and can de-register the victim device from the network.

\section{ACKNOWLEDGMENTS}

We thank anonymous reviewers for providing excellent feedback. This work was partially funded by NSF grant number 2051621 .

\section{REFERENCES}

[1] [n.d.]. IntelliJudge: WaveJudge LTE packets sniffer. http://www.sanjole.com/our-products/intellijudge-lte/.

[2] [n.d.]. QPST- Qualcomm service programmer tool. https://github.com/botletics/SIM7000-LTE-Shield/tree/master/SIM7000\%20Do cumentation/Firmware\%20Updater\%20Tool/QPST\%20Tool.

[3] [n.d.]. QXDM- LTE packets capturing tool.

[4] [n.d.]. Tera-Term-A Terminal Emulator. http://ttssh2.sourceforge.jp/index.html.en.

[5] [n.d.]. ThinkRF: Real-Time Spectrum Analyzer. https://www.thinkrf.com/real-time-spectrum-analyzers/.

[6] 3GPP. 2016. TS24.301: Non-Access-Stratum (NAS) protocol for Evolved Packet System (EPS); Stage 3. http://www.3gpp.org/ftp/Specs/html-info/24301.htm

[7] 3GPP. 2016. TS33.401: 3GPP SAE; Security architecture.

[8] pages $=78-92$ year $=2011$ organization $=$ Springer Aumasson, Jean-Philippe et al., booktitle=International Conference on Information and Communications Security. [n.d.]. A note on a privacy-preserving distance-bounding protocol.

[9] Beurdouche, Benjamin and et al. [n.d.]. A messy state of the union: Taming the composite state machines of TLS. In IEEE Security and Privacy, year=2015.

[10] Böck, Hanno and et al. 2016. Nonce-Disrespecting Adversaries: Practical Forgery Attacks on GCM in TLS. IACR Cryptology ePrint Archive 2016 (2016), 475.

[11] Borisov, Nikita and Goldberg, Ian and Wagner, David. 2001. Intercepting mobile communications: the insecurity of 802.11. In ACM Mobicom.

[12] De Ruiter, Joeri and Poll, Erik. 2015. Protocol State Fuzzing of TLS Implementations.. In USENIX Security Symposium.

[13] Hong, Byeongdo and et al. 2018. GUTI Reallocation Demystified: Cellular Location Tracking with Changing Temporary Identifier. (2018).

[14] Hussain, Syed Rafiul and et al. 2018. LTEInspector: A Systematic Approach for Adversarial Testing of $4 \mathrm{G}$ LTE. In NDSS

[15] Kahn, David. 1996. The codebreakers. New York, NY: Scribner (1996).

[16] Karlof, Chris and Sastry, Naveen and Wagner, David. 2004. TinySec: a link layer security architecture for wireless sensor networks. In ACM SenSys.
[17] Kim, Hongil and et al. 2015. Breaking and fixing volte: Exploiting hidden data channels and mis-implementations. In ACM CCS

[18] $\mathrm{Li}$, Chi-Yu and et al. 2015. Insecurity of voice solution volte in LTE mobile networks. In ACM CCS.

[19] Lichtman, Marc and et al. 2014. Detection and mitigation of uplink control channel jamming in LTE. In IEEE Milcom.

[20] Lichtman, Marc and et al. 2016. LTE/LTE-a jamming, spoofing, and sniffing: threat assessment and mitigation. IEEE Communications Magazine 54, 4 (2016), $54-61$.

[21] Mason, Joshua and et al. 2006. A natural language approach to automated cryptanalysis of two-time pads. In ACM CCS.

[22] MM, Galib Asadullah. 2008. Robust wireless communications under co-channel interference and jamming. Ph.D. Dissertation. Georgia Institute of Technology, $\mathrm{PhD}$ thesis.

[23] Naseef, M. 2014. Vulnerabilities of LTE and LTE-Advanced Communication White Paper. (2014).

[24] Peng, Chunyi and et al. 2012. Mobile data charging: new attacks and countermeasures. In ACM CCS

[25] Peng, Chunyi and et al. 2014. Real threats to your data bills: Security loopholes and defenses in mobile data charging. In ACM CCS.

[26] Qian, Zhiyun and Mao, Z Morley. [n.d.]. Off-path TCP sequence number inference attack-how firewall middleboxes reduce security. In IEEE S\&P.

[27] Raza, Muhammad Taqi and et al. 2017. Exposing LTE Security Weaknesses at Protocol Inter-layer, and Inter-radio Interactions. In SecureComm.

[28] David Rupprecht, Katharina Kohls, Thorsten Holz, and Christina Pöpper. 2020. Call Me Maybe: Eavesdropping Encrypted \{LTE $\}$ Calls With ReVoLTE. In 29th \{USENIX\} Security Symposium (\{USENIX\} Security 20). 73-88.

[29] Rupprecht, David and et al. 2018. Breaking LTE on Layer Two. In IEEE S\&P.

[30] Shaik, Altaf and et al. 2015. Practical attacks against privacy and availability in 4G/LTE mobile communication systems. (2015).

[31] Shannon, CE. 1948. (1948), "A Mathematical Theory of Communication", Bell System Technical Journal, vol. 27, pp. 379-423 \& 623-656, July \& October. (1948).

[32] Stubblefield, Adam and et al. 2004. A key recovery attack on the $802.11 \mathrm{~b}$ wired equivalent privacy protocol (WEP). ACM transactions on information and system security (TISSEC) 7, 2 (2004), 319-332.

[33] Tu, Guan-Hua and et al. 2013. How voice calls affect data in operational LTE networks. In ACM MobiCom.

[34] Tu, Guan-Hua and et al. 2016. Detecting problematic control-plane protocol interactions in mobile networks. IEEE/ACM Transactions on Networking 24,2 (2016), 1209-1222.

[35] Vanhoef, Mathy and Piessens, Frank. 2017. Key reinstallation attacks: Forcing nonce reuse in WPA2. In ACM CCS.

[36] Vaudenay, Serge. 2002. Security Flaws Induced by CBC Padding-Applications to SSL, IPSEC, WTLS.... In International Conference on the Theory and Applications of Cryptographic Techniques. Springer, 534-545.

[37] Vernam, Gilbert Sandford. [n.d.]. Secret signaling system (U, 1919). U.S. Patent 131071 ([n.d.])

[38] Yan, Qiben and et al. 2014. MIMO-based jamming resilient communication in wireless networks. In IEEE Infocom.

[39] Zeng, Huacheng and et al. 2017. Enabling jamming-resistant communications in wireless MIMO networks. In IEEE CNS.

[40] Zenner, Erik. 2009. Nonce generators and the nonce reset problem. In International Conference on Information Security. Springer, 411-426. 